\title{
RECOVERING A PIECEWISE CONSTANT VOLATILITY FROM PERPETUAL PUT OPTION PRICES
}

\author{
BING LU,* Uppsala University
}

\begin{abstract}
In this paper we present a method to recover a time-homogeneous piecewise constant volatility from a finite set of perpetual put option prices. The whole calculation process of the volatility is decomposed into easy computations in many fixed disjoint intervals. In each interval, the volatility is obtained by solving a system of nonlinear equations.

Keywords: Perpetual put option; calibration of models; piecewise constant volatility

2010 Mathematics Subject Classification: Primary 91G20; 60G40
\end{abstract}

\section{Introduction}

One of the most studied problems in mathematical finance is to calculate the price of an option if the diffusion coefficient of the underlying asset is given. In practice, it is often natural to consider the inverse problem: how to compute the volatility of the underlying stock price if a set of option prices is provided. For example, in the classical Black-Scholes model there is a unique correspondence between the constant volatility and the price of a European option. Thus, the implied volatility can be obtained by the Black-Scholes formula if one option price is known. In general, however, if more than one option price is given, a richer model is needed for the underlying process. One such model is the local volatility model, in which the volatility depends on the current stock price and the current time. Also, this model can be calibrated to fit given option data perfectly. Indeed, Dupire [3] showed that the level- and time-dependent volatility can be written in terms of derivatives of European option prices with respect to strike price and maturity.

In the present paper we are interested in the calibration of models from the prices of perpetual American put options. The volatility of the underlying asset is considered to be time homogeneous. Similar to the European case mentioned above, if the Black-Scholes model is offered as the process of the underlying stock price, then it is straightforward to compute the constant volatility if one option price is given. In parallel to Dupire's equation, a leveldependent model for the stock price is given in Ekström and Hobson [4]; see also [1]. Ekström and Hobson assumed that the prices of the perpetual put options are given for all different strike prices and they expressed the diffusion coefficient in terms of the option prices and their derivatives. This volatility is uniquely determined at the price level below the current stock price.

As noted above, both Dupire's formula for the volatility and the level-dependent volatility recovered from prices of perpetual put options are calculated under an assumption of a (possibly double) continuum of given option prices. In reality, however, option prices are only given for

Received 12 October 2009; revision received 19 April 2010.

* Postal address: Department of Mathematics, Uppsala University, Box 480, 75106 Uppsala, Sweden.

Email address: bing@math.uu.se 
a discrete set of strike prices, so one then needs to interpolate between them. Moreover, since the volatility is calculated using derivatives of the option prices, it is very sensitive to the interpolation procedure. On the other hand, the constant volatility defined in the Black-Scholes model is easy to calculate, but generically it is impossible to fit one constant volatility if several option prices are given.

Motivated by the discussion above, we consider a situation in which prices of perpetual American put options are given for a finite set of strike prices. To rule out arbitrage possibilities, the option price has to be increasing and convex in the strike. Moreover, we assume that the option price is strictly convex in strikes. Since there does not exist a continuum of option prices, we can create plenty of time-homogeneous models to reproduce the option data (one for each choice of interpolation procedure). A natural candidate for the time-homogeneous volatility model is the piecewise constant function of the stock price. In the present paper we prove the existence of a piecewise constant volatility that reproduces the given option prices. Given $n$ option prices, the whole calculation process is decomposed into an elementary computation in $n$ fixed disjoint intervals. To obtain the volatility in each interval, we just need to solve two nonlinear equations with two unknown variables. Moreover, since it does not involve differentiation of the option price, we believe that it is more stable with respect to small changes in the input than the model by Ekström and Hobson.

The paper is organized as follows. In Section 2 we study the forward problem. Provided that the volatility is a piecewise constant function of the underlying stock price, we can calculate the price of the perpetual put option for different strike prices. Section 3 treats the inverse problem and contains our main results. Given a finite set of prices of the perpetual put options, we present a method to construct a piecewise constant volatility which reproduces the option prices. In Section 4, the results are illustrated using a numerical example.

\section{The forward problem}

We consider a model where the process of the stock price $X$ solves the stochastic differential equation

$$
\mathrm{d} X_{t}=r X_{t} \mathrm{~d} t+\sigma\left(X_{t}\right) X_{t} \mathrm{~d} W_{t} .
$$

Here $r$ is the constant interest rate, $W$ is the standard Brownian motion, and $\sigma\left(X_{t}\right)$ is a positive function. Given the current stock price $x_{0}$, the price of a perpetual American put option with strike $K$ is

$$
P(K)=\sup _{\tau} \mathrm{E}^{x_{0}}\left[\mathrm{e}^{-r \tau}\left(K-X_{\tau}\right)^{+}\right],
$$

where $\tau$ is any stopping time with respect to the filtration generated by $W$. The solution to the optimal stopping problem (1) is closely related to the ordinary differential equation (ODE)

$$
\frac{1}{2} \sigma^{2}(x) x^{2} u_{x x}+r x u_{x}-r u=0 .
$$

There are two linearly independent solutions to this ODE. If one of them is positive and increasing and the other one positive and decreasing, then they are unique up to positive multiplicative constants; cf. [2, pp. 18-19]. We denote these solutions by $\psi$ and $\varphi$, respectively. Without loss of generality, we choose the decreasing solution to satisfy $\varphi\left(x_{0}\right)=1$. Define the hitting times $H_{z}=\inf \left\{t \geq 0: X_{t}=z\right\}$. Since $\mathrm{e}^{-r t} \varphi\left(X_{t}\right)$ is a local martingale and $\varphi(x)$ is decreasing in $x$, we have

$$
\mathrm{E}^{x}\left[\mathrm{e}^{-r H_{z}}\right]=\frac{\varphi(x)}{\varphi(z)} \quad \text { if } x>z .
$$




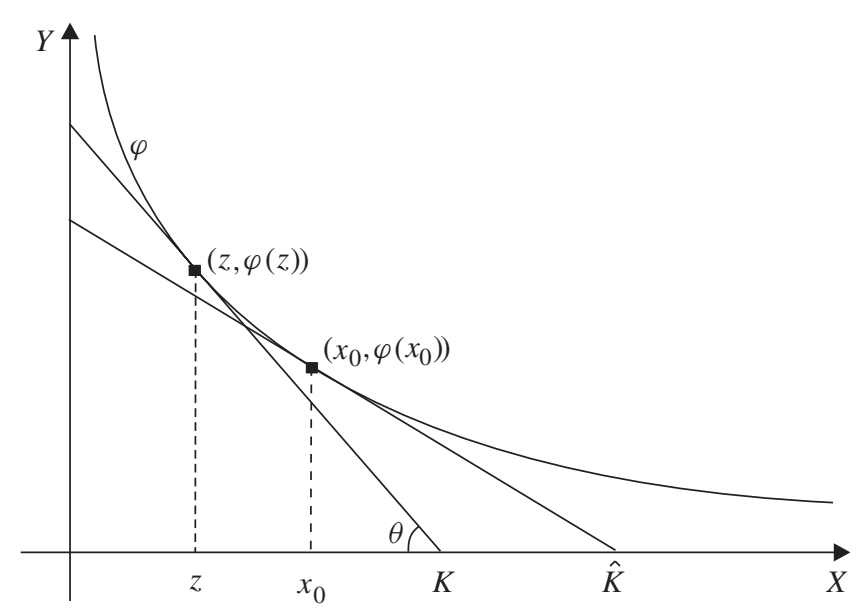

FIGURE 1: The picture shows how to determine the optimal exercise level $z$ for a given strike $K$.

Owing to the time homogeneity of problem (1), it suffices to take the supremum over stopping times that are exit times from an interval. Moreover, since put options are considered, we only need to take the supremum over hitting times $H_{z}$ for some level $z$; cf. [4, Proof of Lemma 2.2]. We thus find that

$$
\begin{aligned}
P(K) & =\sup _{\tau} \mathrm{E}^{x_{0}}\left[\mathrm{e}^{-r \tau}\left(K-X_{\tau}\right)^{+}\right] \\
& =\sup _{\left\{z: z \leq x_{0} \wedge K\right\}} \mathrm{E}^{x_{0}}\left[\mathrm{e}^{-r H_{z}}\left(K-X_{H_{z}}\right)^{+}\right] \\
& =\sup _{\left\{z: z \leq x_{0} \wedge K\right\}}(K-z) \mathrm{E}^{x_{0}}\left[\mathrm{e}^{-r H_{z}}\right] \\
& =\sup _{\left\{z: z \leq x_{0}\right\}} \frac{K-z}{\varphi(z)} .
\end{aligned}
$$

It is easy to check that the function $\varphi(z)$ is strictly convex and decreasing in $z$. In Figure 1, $\theta$ denotes the acute angle between the $x$-axis and the line passing through $(K, 0)$ and $(z, \varphi(z))$. Here $z$ can be any price level below $x_{0}$ and $K$. It is easy to see that $(K-z) / \varphi(z)=\cot (\theta)$. Thus, we just need to find the smallest $\theta$ for $z \leq x_{0}$. Define

$$
\hat{K}=x_{0}-\frac{\varphi\left(x_{0}\right)}{\varphi^{\prime}\left(x_{0}\right)}=x_{0}-\frac{1}{\varphi^{\prime}\left(x_{0}\right)},
$$

so that the line passing through $(\hat{K}, 0)$ and $\left(x_{0}, \varphi\left(x_{0}\right)\right)$ is tangent to the curve $\varphi$.

When $K \leq \hat{K}$, it is not optimal to exercise the option immediately. Instead, the investors should wait until the stock price hits the optimal stopping level to exercise the option. Thus, we have

$$
P(K)=\sup _{z} \frac{K-z}{\varphi(z)},
$$

where the optimal $z$, which is the optimal exercise level for strike $K$, is chosen so that

$$
(K-z) \varphi^{\prime}(z)+\varphi(z)=0 .
$$


Equation (4) indicates that the straight line passing through $(K, 0)$ and $(z, \varphi(z))$ is tangent to the function $\varphi$. We also obtain $P(K)=K-x_{0}$ for $K>\hat{K}$, which implies that it is optimal for investors to exercise the option immediately.

We now specialize to the case of piecewise constant volatility. More precisely, it is assumed that the interval $\left[0, x_{0}\right]$ is divided by a mesh consisting of $n$ points $a_{1}, a_{2}, a_{3}, \ldots, a_{n}$, which satisfy $0<a_{1}<a_{2}<\cdots<a_{n-1}<a_{n} \leq x_{0}$. The volatility function $\sigma\left(X_{t}\right)$ is defined as

$$
\sigma(x)= \begin{cases}\sigma_{0}, & 0<x<a_{1} \\ \sigma_{i}, & a_{i} \leq x<a_{i+1}, 1 \leq i \leq n-1, \\ \sigma_{n}, & a_{n} \leq x\end{cases}
$$

where $\sigma_{0}, \ldots, \sigma_{n}$ are positive constants. On an interval $\left(a_{i}, a_{i+1}\right)$, the volatility is constant and therefore the fundamental solution $\varphi$ is $C^{\infty}$. However, at a jump point $a_{i}$ of $\sigma$, the function $\varphi$ is merely $C^{1}$ and the second derivative has a jump. Given the piecewise constant volatility function $\sigma(x)$ defined above, the two independent positive solutions of the ODE (2) are

$$
\psi(x)=x
$$

and

$$
\varphi(x)= \begin{cases}A_{0} x^{-\beta_{0}}+B_{0} x, & 0<x<a_{1}, \\ A_{i} x^{-\beta_{i}}+B_{i} x, & a_{i} \leq x<a_{i+1}, 1 \leq i \leq n-1, \\ A_{n} x^{-\beta_{n}}+B_{n} x, & a_{n} \leq x,\end{cases}
$$

where $\beta_{i}=2 r / \sigma_{i}^{2}$ for $i \in\{0, \ldots, n\}$. Here $A_{i}$ and $B_{i}$ for $i \in\{0, \ldots, n\}$ are chosen so that $\varphi(x)$ is $C^{1}$ everywhere. Without loss of generality, we let $\varphi\left(x_{0}\right)=1$; thus,

$$
A_{n}=x_{0}^{\beta_{n}}, \quad B_{n}=0,
$$

since $\varphi(x)$ is decreasing and nonnegative for $x \geq a_{n}$. Owing to the $C^{1}$-regularity, we have

$$
\begin{aligned}
A_{i} a_{i+1}^{-\beta_{i}}+B_{i} a_{i+1} & =A_{i+1} a_{i+1}^{-\beta_{i+1}}+B_{i+1} a_{i+1}, \\
-A_{i} \beta_{i} a_{i+1}^{-\beta_{i}-1}+B_{i} & =-A_{i+1} \beta_{i+1} a_{i+1}^{-\beta_{i+1}-1}+B_{i+1} .
\end{aligned}
$$

It follows that

$$
A_{i}=A_{i+1} a_{i+1}^{\beta_{i}-\beta_{i+1}} \frac{1+\beta_{i+1}}{1+\beta_{i}}, \quad B_{i}=A_{i+1} a_{i+1}^{-\beta_{i+1}-1} \frac{\beta_{i}-\beta_{i+1}}{1+\beta_{i}}+B_{i+1},
$$

for $i \in\{0, \ldots, n-1\}$.

The function $\varphi(x)$ defined in (6), (7), and (8) is the decreasing fundamental solution to the ODE (2). Hence, for $K \leq \hat{K}=x_{0}\left(1+1 / \beta_{n}\right)$, the option price is

$$
P(K)=\sup _{z} \frac{K-z}{\varphi(z)}
$$

where the optimal $z$ is determined by

$$
(K-z) \varphi^{\prime}(z)+\varphi(z)=0 .
$$

For $K>\hat{K}$, we have $P(K)=K-x_{0}$. Since $\varphi(x)$ is $C^{1}$ and strictly convex in $x$, (10) defines a one-to-one correspondence between strike prices $K \in(0, \hat{K}]$ and optimal exercise 
levels $z \in\left(0, x_{0}\right]$. Now let $K_{i}^{*}$ be the strike price for which $a_{i}$ is the optimal exercise level. By (10) we have

$$
K_{i}^{*}=-\frac{\varphi\left(a_{i}\right)}{\varphi^{\prime}\left(a_{i}\right)}+a_{i}=\frac{\left(1+\beta_{i}\right) A_{i}}{\beta_{i} A_{i} a_{i}^{-1}-B_{i} a_{i}^{\beta_{i}}}
$$

for $1 \leq i \leq n$. It is straightforward to find that

$$
K_{n}^{*}=\frac{a_{n}\left(1+\beta_{n}\right)}{\beta_{n}} .
$$

Since $K$ is strictly increasing as a function of $z, K_{i}^{*}$ is increasing in $i$. Moreover, for $K \in$ $\left[K_{i}^{*}, K_{i+1}^{*}\right.$ ), the optimal exercise level $z$ belongs to $\left[a_{i}, a_{i+1}\right)$. By summarizing all our findings in this section we obtain the following theorem.

Theorem 1. Given the piecewise constant volatility defined in (5), the price of the perpetual American put option defined by (1) is given by

$$
P(K)= \begin{cases}K-x_{0}, & K \geq \hat{K}, \\ \frac{K^{\beta_{n}+1} \beta_{n}^{\beta_{n}}}{x_{0}^{\beta_{n}}\left(1+\beta_{n}\right)^{1+\beta_{n}}}, & K_{n}^{*} \leq K<\hat{K}, \\ \frac{K-z}{A_{i} z^{-\beta_{i}+B_{i} z},} & K_{i}^{*} \leq K<K_{i+1}^{*}, 1 \leq i \leq n-1, \\ \frac{K-z}{A_{0} z^{-\beta_{0}+B_{0} z},} & 0<K<K_{1}^{*} .\end{cases}
$$

Here $A_{i}$ and $B_{i}$ for $i \in\{0, \ldots, n-1\}$ are defined by (8). The optimal exercise level $z$ in (11) is determined implicitly by

$$
(K-z)\left(-A_{i} \beta_{i} z^{-\beta_{i}-1}+B_{i}\right)+A_{i} z^{-\beta_{i}}+B_{i} z=0
$$

if $K \in\left[K_{i}^{*}, K_{i+1}^{*}\right)$ for $i \in\{1, \ldots, n-1\}$ or if $K<K_{1}^{*}$ for $i=0$.

By Theorem 1, the option price $P(K)$ can be computed explicitly if a piecewise constant volatility $\sigma(x)$ is given as in (5).

\section{The inverse problem}

In this section we take the point of view that option prices for a discrete set of strikes written on a certain underlying asset can be recorded from the market. We construct a piecewise constant volatility function of the underlying stock price, which is calibrated to perfectly fit the finite set of option prices.

Assume that $n$ strike prices and the corresponding $n$ perpetual put option prices are given from the market data. Arbitrage considerations show that the put option price has to be nondecreasing and convex in $K$. Below we make the slightly stronger assumptions that it is increasing and strictly convex. Thus, the option price $P\left(K_{i}\right)$ has to satisfy

$$
P\left(K_{1}\right)<P\left(K_{2}\right)<\cdots<P\left(K_{n}\right)
$$

for the strike prices $0<K_{1}<K_{2}<\cdots<K_{n}$. For the index level $n$, we assume that $K_{n}$ satisfies $P\left(K_{n}\right)=K_{n}-x_{0}$, where $x_{0}$ is the current stock price. Below $K_{n}$ will correspond 
to $\hat{K}$ in the forward problem, so any option with strike price that is bigger than or equal to $K_{n}$ should be exercised immediately. Later in this section we will discuss the case when $K_{n}$ cannot be observed from the market. We also assume that the natural bounds

$$
\left(K_{i}-x_{0}\right)^{+}<P\left(K_{i}\right)<K_{i}
$$

for $i \in\{1, \ldots, n-1\}$ are fulfilled, where the first strict inequality implies that it is not optimal to exercise the option immediately. We also assume that the value function $P(K)$ is strictly convex in the strikes, so that

$$
\frac{P\left(K_{2}\right)-P\left(K_{1}\right)}{K_{2}-K_{1}}>\frac{P\left(K_{1}\right)}{K_{1}}
$$

and

$$
\frac{P\left(K_{i}\right)-P\left(K_{i-1}\right)}{K_{i}-K_{i-1}}<\frac{P\left(K_{i+1}\right)-P\left(K_{i}\right)}{K_{i+1}-K_{i}}
$$

for $i \in\{2, \ldots, n-1\}$. It follows that

$$
\frac{P\left(K_{i}\right)-P\left(K_{i-1}\right)}{K_{i}-K_{i-1}}<1
$$

for $i \in\{2, \ldots, n\}$.

Next we will draw a graph containing all the information given by (13), (14), (15), (16), and (17). In the $x-\varphi(x)$ coordinate system, draw the lines passing through $\left(K_{i}, 0\right)$ with slope $-1 / P\left(K_{i}\right)$ for every $i \in\{1, \ldots, n\}$. We refer to these lines as 'option lines' and denote the option line with index level $i$ by $l_{i}$. According to (16), we have

$$
\frac{K_{i}-K_{i-1}}{P\left(K_{i}\right)-P\left(K_{i-1}\right)}>\frac{K_{i+1}-K_{i}}{P\left(K_{i+1}\right)-P\left(K_{i}\right)},
$$

where the expression on the left-hand side is the second coordinate of the intersection between $l_{i}$ and $l_{i-1}$, and the expression on the right-hand side is the second coordinate of the intersection between $l_{i+1}$ and $l_{i}$.

According to (15), we obtain

$$
\frac{K_{2}-K_{1}}{P\left(K_{2}\right)-P\left(K_{1}\right)}<\frac{K_{1}}{P\left(K_{1}\right)},
$$

which implies that the second coordinate of the intersection between $l_{2}$ and $l_{1}$ is smaller than the second coordinate of the intersection between $l_{1}$ and and $\varphi(x)$-axis. Therefore, as $i$ decreases from $n$ to 2 , the first and second coordinates of the intersection between $l_{i}$ and $l_{i-1}$ are all positive, and decrease and increase, respectively. Equation (14) gives

$$
\frac{K_{i}-x_{0}}{P\left(K_{i}\right)}<1
$$

for any $i \in\{1, \ldots, n-1\}$, which implies that $\left(x_{0}, 1\right)$ is on the right side of all the option lines except $l_{n}$. Note that the point $\left(x_{0}, 1\right)$ is on $l_{n}$. According to (17), we have

$$
\frac{K_{n}-K_{n-1}}{P\left(K_{n}\right)-P\left(K_{n-1}\right)}>1 \text {. }
$$

This shows that the second coordinate of the intersection between $l_{n}$ and $l_{n-1}$ is larger than 1 . Summing up all the information mentioned above, Figure 2 gives a simple version of option lines. 


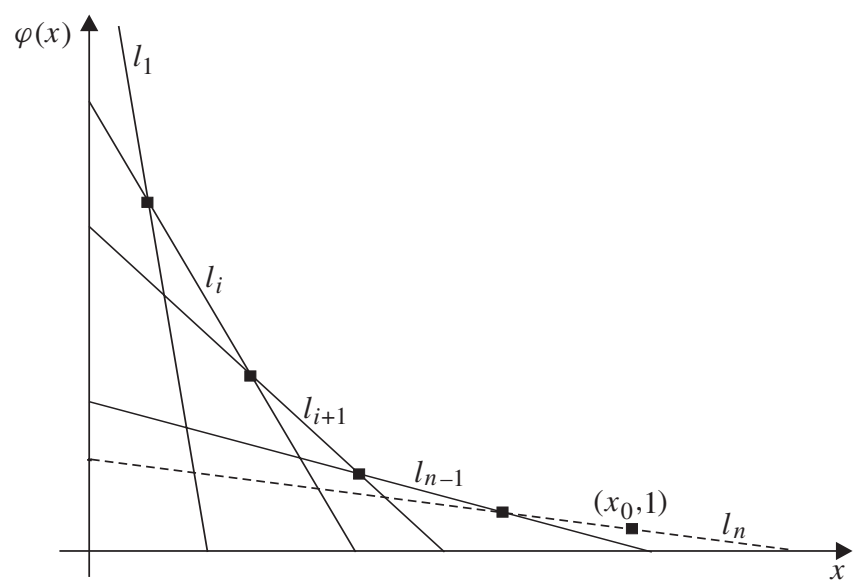

Figure 2: The option line $l_{i}$ has slope $-1 / P\left(K_{i}\right)$ and intersects the $x$-axis at the point $\left(K_{i}, 0\right)$.

Theorem 2. Assume that $n$ strike prices $K_{1}, \ldots, K_{n}$ and the corresponding prices of perpetual put options $P\left(K_{1}\right), \ldots, P\left(K_{n}\right)$ satisfying conditions (13), (14), (15), (16), (17) and $P\left(K_{n}\right)=$ $K_{n}-x_{0}$ are given, where $x_{0}$ is the current stock price. Then there exists a time-homogeneous process with a piecewise constant volatility that recovers the option prices.

\subsection{Proof of Theorem 2}

We are looking for a piecewise constant volatility of the form

$$
\sigma(x)= \begin{cases}\sigma_{0}, & 0<x<b_{1} \\ \sigma_{i 1}, & b_{i} \leq x<c_{i}, 1 \leq i \leq n-1 \\ \sigma_{i 2}, & c_{i} \leq x<b_{i+1}, 1 \leq i \leq n-1 \\ \sigma^{*}, & x \geq b_{n}=x_{0}\end{cases}
$$

where $0<b_{1}<c_{1}<\cdots<b_{i}<c_{i}<b_{i+1}<\cdots<b_{n}=x_{0}$. With this volatility, the decreasing fundamental solution to the ODE (2) is of the form

$$
\varphi(x)= \begin{cases}A_{0} x^{-\beta_{0}}+B_{0} x, & 0<x<b_{1} \\ A_{i 1} x^{-\beta_{i 1}}+B_{i 1} x, & b_{i} \leq x<c_{i}, 1 \leq i \leq n-1 \\ A_{i 2} x^{-\beta_{i 2}}+B_{i 2} x, & c_{i} \leq x<b_{i+1}, 1 \leq i \leq n-1, \\ A^{*} x^{-\beta^{*}+B^{*} x,} & x_{0} \leq x\end{cases}
$$

where $\beta_{0}=2 r / \sigma_{0}^{2}$ (and similarly for $\beta_{i 1}, \beta_{i 2}$, and $\beta^{*}$ ). The constants $A_{0}, A_{i 1}, A_{i 2}, A^{*}, B_{0}$, $B_{i 1}, B_{i 2}$, and $B^{*}$ should be chosen so that $\varphi(x)$ is $C^{1}$ everywhere. If we can find a function $\varphi(x)$ of the form (19) with suitable parameters which is tangent to all the option lines and touching the point $\left(x_{0}, 1\right)$, and the first coordinates of these tangent points are not bigger than $x_{0}$, then it satisfies

$$
P\left(K_{i}\right)=\sup _{z} \frac{K_{i}-z}{\varphi(z)},
$$

and the optimal $z$ for each $K_{i}$ is smaller than or equal to $x_{0}$. Hence, the corresponding piecewise constant volatility $\sigma(x)$ is calibrated to fit the set of option prices perfectly and is the volatility that we are looking for. 
Next we determine $b_{i}$ for $i \in\{1, \ldots, n\}$ and $c_{i}$ for $i \in\{1, \ldots, n-1\}$.

Let $d_{i}$ be the first coordinate of the intersection of $l_{i}$ and $l_{i+1}$. Thus, we have

$$
d_{i}=\frac{K_{i} P\left(K_{i+1}\right)-K_{i+1} P\left(K_{i}\right)}{P\left(K_{i+1}\right)-P\left(K_{i}\right)}
$$

for $i \in\{1, \ldots, n-1\}$. Choose $b_{i}$ to be

$$
b_{i}=\frac{d_{i-1}+d_{i}}{2}
$$

for $i \in\{2, \ldots, n-1\}$, and $b_{1}=d_{1} / 2$ and $b_{n}=x_{0}$. We will construct $\varphi$ so that $b_{i}$ is the first coordinate of the tangent point where $\varphi$ touches the $l_{i}$. (In fact, this tangent point could be chosen anywhere on the segment that connects the nearest two intersections, but we believe that the mid-point is a natural choice. For $b_{1}$, one may argue that $b_{1}=2 d_{1}-b_{2}$ is another natural choice in some cases.) The second coordinate of the tangent point corresponding to $b_{i}$ is

$$
\begin{aligned}
\varphi\left(b_{i}\right)= & \frac{\left(K_{i}-K_{i-1}\right)\left(P\left(K_{i+1}\right)-P\left(K_{i}\right)\right)}{2\left(P\left(K_{i}\right)-P\left(K_{i-1}\right)\right)\left(P\left(K_{i+1}\right)-P\left(K_{i}\right)\right)} \\
& +\frac{\left(K_{i+1}-K_{i}\right)\left(P\left(K_{i}\right)-P\left(K_{i-1}\right)\right)}{2\left(P\left(K_{i}\right)-P\left(K_{i-1}\right)\right)\left(P\left(K_{i+1}\right)-P\left(K_{i}\right)\right)}
\end{aligned}
$$

for $i \in\{2, \ldots, n-1\}$, which can be easily computed by plugging in the option data. Additionally, we have

$$
\varphi\left(b_{1}\right)=\frac{2 K_{1}-d_{1}}{2 P\left(K_{1}\right)}, \quad \varphi\left(b_{n}\right)=1 .
$$

For simplicity, we let $c_{i}$ equal $d_{i}$ defined in (20). For a graphic illustration of the choices of $b_{i}$ and $c_{i}$, see Figure 3 .

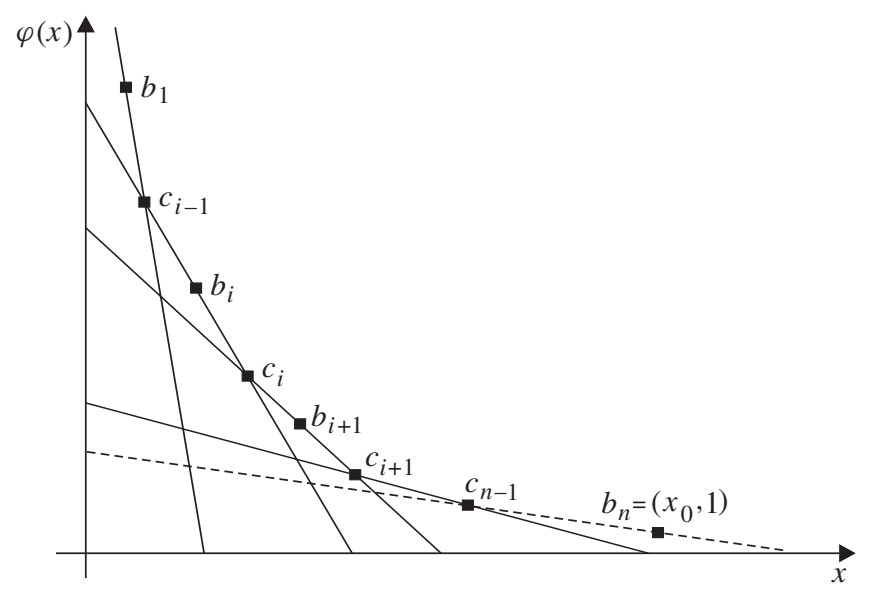

FIGURE 3: The break point $c_{i}$ is chosen as the first coordinate of the intersection between $l_{i}$ and $l_{i+1}$. The break point $b_{i}$ is chosen as the average of $c_{i-1}$ and $c_{i}$. 
In the interval $\left[b_{i}, b_{i+1}\right)$ for $i \in\{1, \ldots, n-1\}$ the function $\varphi(x)$ should be $C^{1}$, which implies the following equations:

$$
\begin{aligned}
\varphi\left(b_{i}\right) & =A_{i 1} b_{i}^{-\beta_{i 1}}+B_{i 1} b_{i}, \\
\left.\frac{\partial \varphi(x)}{\partial x}\right|_{x=b_{i}} & =-\beta_{i 1} A_{i 1} b_{i}^{-\beta_{i 1}-1}+B_{i 1}=-\frac{1}{P\left(K_{i}\right)}, \\
\varphi\left(b_{i+1}\right) & =A_{i 2} b_{i+1}-\beta_{i 2}+B_{i 2} b_{i+1}, \\
\left.\frac{\partial \varphi(x)}{\partial x}\right|_{x=b_{i+1}} & =-\beta_{i 2} A_{i 2} b_{i+1}-\beta_{i 2}-1+B_{i 2}=-\frac{1}{P\left(K_{i+1}\right)}, \\
\varphi\left(c_{i}\right) & =A_{i 1} c_{i}^{-\beta_{i 1}}+B_{i 1} c_{i}=A_{i 2} c_{i}^{-\beta_{i 2}}+B_{i 2} c_{i}, \\
\left.\frac{\partial \varphi(x)}{\partial x}\right|_{x=c_{i}} & =-\beta_{i 1} A_{i 1} c_{i}^{-\beta_{i 1}-1}+B_{i 1}=-\beta_{i 2} A_{i 2} c_{i}^{-\beta_{i 2}-1}+B_{i 2} .
\end{aligned}
$$

Let $\hat{\varphi}\left(x, \beta_{i 1}\right)=\varphi(x)$ be a function of the variables $x$ and $\beta_{i 1}$ for $x \in\left[b_{i}, c_{i}\right]$, and let $\hat{\varphi}\left(x, \beta_{i 2}\right)=$ $\varphi(x)$ be a function of the variables $x$ and $\beta_{i 2}$ for $x \in\left[c_{i}, b_{i+1}\right)$. Note that $\varphi\left(b_{i}\right)$ and $\varphi\left(b_{i+1}\right)$ are constants that we can compute, $\varphi(x)$ is a function of the variable $x, \varphi\left(x, \beta_{i 1}\right)$ is a function with variables $x$ and $\beta_{i 1}$, and $\varphi\left(x, \beta_{i 2}\right)$ is a function with variables $x$ and $\beta_{i 2}$. After some manipulation the equations become

$$
\begin{aligned}
& \hat{\varphi}\left(x, \beta_{i 1}\right)= \frac{\varphi\left(b_{i}\right) P\left(K_{i}\right)+b_{i}}{\left(1+\beta_{i 1}\right) P\left(K_{i}\right)}\left(\frac{b_{i}}{x}\right)^{\beta_{i 1}}+\frac{P\left(K_{i}\right) \beta_{i 1} \varphi\left(b_{i}\right)-b_{i}}{P\left(K_{i}\right)\left(1+\beta_{i 1}\right)} \frac{x}{b_{i}}, \quad b_{i} \leq x<c_{i}, \\
& \hat{\varphi}\left(x, \beta_{i 2}\right)= \frac{\varphi\left(b_{i+1}\right) P\left(K_{i+1}\right)+b_{i+1}}{\left(1+\beta_{i 2}\right) P\left(K_{i+1}\right)}\left(\frac{b_{i+1}}{x}\right)^{\beta_{i 2}} \\
&+\frac{P\left(K_{i+1}\right) \beta_{i 2} \varphi\left(b_{i+1}\right)-b_{i+1}}{P\left(K_{i+1}\right)\left(1+\beta_{i 2}\right)} \frac{x}{b_{i+1}}, \quad c_{i} \leq x \leq b_{i+1}, \\
& \hat{\varphi}\left(c_{i}, \beta_{i 1}\right)= \hat{\varphi}\left(c_{1}, \beta_{i 2}\right), \\
&\left.\frac{\partial \hat{\varphi}\left(x, \beta_{i 1}\right)}{\partial x}\right|_{x=c_{i}}=\left.\frac{\partial \hat{\varphi}\left(x, \beta_{i 2}\right)}{\partial x}\right|_{x=c_{i}},
\end{aligned}
$$

for $i \in\{1, \ldots, n-1\}$. The option prices $P\left(K_{i}\right)$ and $K_{i}$ for $i \in\{1, \ldots, n\}$ are given, and it is straightforward to compute $c_{i}, b_{i}$, and $\varphi\left(b_{i}\right)$ for each $i$, so in each interval $\left[b_{i}, b_{i+1}\right)$ there are only two unknown parameters $\beta_{i 1}$ and $\beta_{i 2}$ to be determined. Next we will prove the existence of a solution to the system of equations (24a)-(24d).

It is easy to check that

$$
\lim _{\beta_{i 1} \rightarrow 0} \hat{\varphi}\left(x, \beta_{i 1}\right)=\varphi\left(b_{i}\right)-\frac{x-b_{i}}{P\left(K_{i}\right)},
$$

which implies that $\hat{\varphi}\left(x, \beta_{i 1}\right)$ tends to $l_{i}$ as $\beta_{i 1}$ goes to 0 . Similarly, we can show that $\hat{\varphi}\left(x, \beta_{i 2}\right)$ tends to $l_{i+1}$ as $\beta_{i 2}$ goes to 0 . We can also check that

$$
\lim _{\beta_{i 1} \rightarrow \infty} \hat{\varphi}\left(x, \beta_{i 1}\right)=\frac{\varphi\left(b_{i}\right) x}{b_{i}}, \quad \lim _{\beta_{i 2} \rightarrow \infty} \hat{\varphi}\left(x, \beta_{i 2}\right) \rightarrow \infty .
$$

Claim 1. The functions $\hat{\varphi}\left(x, \beta_{i 1}\right)$ and $\hat{\varphi}\left(x, \beta_{i 2}\right)$ are increasing in $\beta_{i 1}$ and $\beta_{i 2}$, respectively. 
Proof. For $x \in\left[b_{i}, c_{i}\right)$, define $z=b_{i} / x$. Then

$$
\hat{\varphi}\left(x, \beta_{i 1}\right)=\frac{\varphi\left(b_{i}\right) P\left(K_{i}\right)+b_{i}}{\left(1+\beta_{i 1}\right) P\left(K_{i}\right)} z^{\beta_{i 1}}+\frac{P\left(K_{i}\right) \beta_{i 1} \varphi\left(b_{i}\right)-b_{i}}{z P\left(K_{i}\right)\left(1+\beta_{i 1}\right)} .
$$

Taking the derivative of $\hat{\varphi}\left(x, \beta_{i 1}\right)$ with respect to $\beta_{i 1}$ yields

$$
\frac{1+\beta_{i 1}}{\varphi\left(b_{i}\right)+b_{i} / P\left(K_{i}\right)} \frac{\partial \hat{\varphi}\left(x, \beta_{i 1}\right)}{\partial \beta_{i 1}}=z^{\beta_{i 1}} \ln z+\frac{1}{1+\beta_{i 1}}\left(\frac{1}{z}-z^{\beta_{i 1}}\right)=f(z) .
$$

Note that $0<z \leq 1$ and $f(1)=0$. Thus, in order to show that $\hat{\varphi}\left(x, \beta_{i 1}\right)$ is increasing in $\beta_{i 1}$, it suffices to show that $f(z)$ is positive for $0<z<1$. Next, differentiating $z f(z)$ with respect to $z$ gives

$$
\frac{\partial(z f(z))}{\partial z}=\left(1+\beta_{i 1}\right) z^{\beta_{i 1}} \ln z<0
$$

for $0<z<1$. Note that $1 \times f(1)=0$; thus, $z f(z)>0$ for $0<z<1$. It follows that $f(z)>0$ for $0<z<1$, which implies that $\partial \varphi\left(x, \beta_{i 1}\right) / \partial \beta_{i 1}>0$. We can also show that $\partial \varphi\left(x, \beta_{i 2}\right) / \partial \beta_{i 2}>0$ by a similar argument as above. This completes the proof of the claim.

Therefore, as $\beta_{i 1}$ increases from 0 to $\infty, \hat{\varphi}\left(x, \beta_{i 1}\right)$ increases from $\varphi\left(b_{i}\right)-\left(x-b_{i}\right) / P\left(K_{i}\right)$ to $\varphi\left(b_{i}\right) x / b_{i}$. Again, as $\beta_{i 2}$ increases from 0 to $\infty, \varphi\left(x, \beta_{i 2}\right)$ also increases from $\varphi\left(b_{i+1}\right)-$ $\left(x-b_{i+1}\right) / P\left(K_{i+1}\right)$ to $\infty$. Let $A$ be the point $\left(c_{i},\left(K_{i}-c_{i}\right) / P\left(K_{i}\right)\right)$, and let $B$ be the point

$$
\left(c_{i}, \frac{\varphi\left(b_{i}\right)\left(b_{i+1}-c_{i}\right)+\varphi\left(b_{i+1}\right)\left(c_{i}-b_{i}\right)}{b_{i+1}-b_{i}}\right)
$$

cf. Figure 4. If we choose a point on the segment $A B$, except the point $A$, then there exist $\beta_{i 1}$ and $\beta_{i 2}$ such that $\hat{\varphi}\left(x, \beta_{i 1}\right)$ and $\hat{\varphi}\left(x, \beta_{i 2}\right)$ pass through that point. For $\beta_{i 1}$ and $\beta_{i 2}$ sufficiently small, $\hat{\varphi}\left(x, \beta_{i 1}\right)$ and $\hat{\varphi}\left(x, \beta_{i 2}\right)$ will meet at some point at the segment $A B$ which is very close to the point $A$. Clearly, the angle between $\hat{\varphi}\left(x, \beta_{i 1}\right)$ and $\hat{\varphi}\left(x, \beta_{i 2}\right)$ is smaller than 180; cf. Figure 4. If $\hat{\varphi}\left(x, \beta_{i 1}\right)$ and $\hat{\varphi}\left(x, \beta_{i 2}\right)$ meet at point $B$, the angle between the two $\hat{\varphi}$ functions is larger than $180^{\circ}$ due to the convexity of $\hat{\varphi}(x, \cdot)$ in $x$. As the intersection of the two $\hat{\varphi}$ functions moves from $A$ to $B$ along the segment $A B$, the angle between the

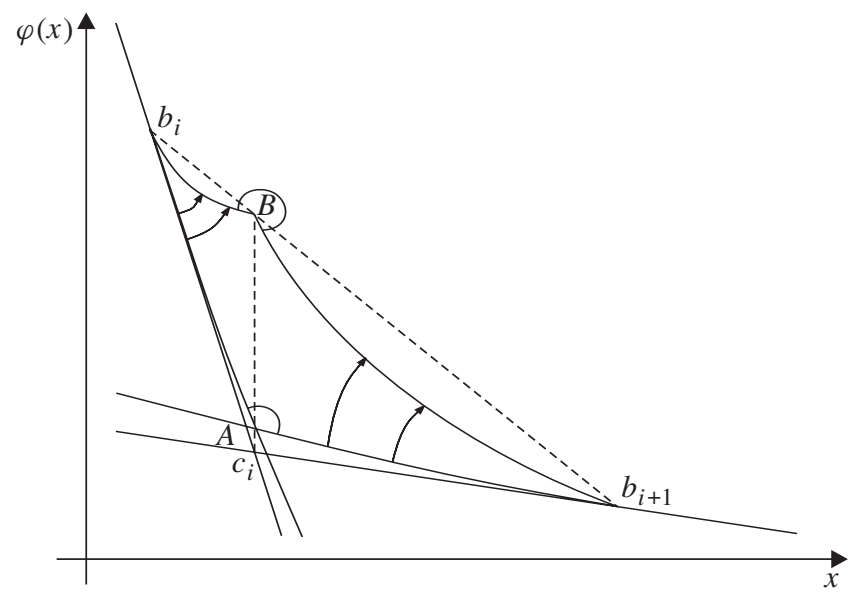

FIGURE 4: The existence of $\beta_{i 1}$ and $\beta_{i 2}$. 
two $\hat{\varphi}$ functions increases from less than $180^{\circ}$ to more than $180^{\circ}$. Hence, by a continuity argument, there exists a point on the segment $A B$ such that the angle between the two $\hat{\varphi}$ functions is exactly $180^{\circ}$. For this particular choice of $\beta_{i 1}$ and $\beta_{i 2}$, define $\varphi$ by $\varphi(x)=$ $\hat{\varphi}\left(x, \beta_{i 1}\right)$ for $x \in\left[b_{i}, c_{i}\right]$ and $\varphi(x)=\hat{\varphi}\left(x, \beta_{i 2}\right)$ for $x \in\left[c_{i}, b_{i+1}\right)$. In this way, $\varphi$ is $C^{1}$ at $c_{i}$.

This completes the proof of the existence of $\beta_{i 1}$ and $\beta_{i 2}$ for $i \in\{1, \ldots, n-1\}$.

Note that $\beta_{0}$ only influences the prices of options with $K<K_{1}$ which are not given. Thus, $\beta_{0}$ can be defined arbitrarily. For simplicity, we let $\beta_{0}=\beta_{11}$. It follows that $A_{0}=A_{11}$ and $B_{0}=B_{11}$. Since $\varphi(x)$ is $C^{1}$ and decreasing in $x$ and $\varphi\left(x_{0}\right)=1$, it is easy to calculate that $\beta^{*}=x_{0} / P\left(K_{n}\right), B^{*}=0$, and $A^{*}=x_{0} \beta^{*}$. This completes the proof of Theorem 2 .

According to (24a)-(24d), in the interval $\left[b_{i}, b_{i+1}\right)$ for $i \in\{1, \ldots, n-1\}$, we just need to solve the two nonlinear equations

$$
\begin{aligned}
& \frac{\varphi\left(b_{i}\right) P\left(K_{i}\right)+b_{i}}{\left(1+\beta_{i 1}\right) P\left(K_{i}\right)}\left(\frac{b_{i}}{c_{i}}\right)^{\beta_{i 1}}+\frac{P\left(K_{i}\right) \beta_{i 1} \varphi\left(b_{i}\right)-b_{i}}{P\left(K_{i}\right)\left(1+\beta_{i 1}\right)} \frac{c_{i}}{b_{i}} \\
& =\frac{\varphi\left(b_{i+1}\right) P\left(K_{i+1}\right)+b_{i+1}}{\left(1+\beta_{i 2}\right) P\left(K_{i+1}\right)}\left(\frac{b_{i+1}}{c_{i}}\right)^{\beta_{i 2}}+\frac{P\left(K_{i+1}\right) \beta_{i 2} \varphi\left(b_{i+1}\right)-b_{i+1}}{P\left(K_{i+1}\right)\left(1+\beta_{i 2}\right)} \frac{c_{i}}{b_{i+1}}
\end{aligned}
$$

and

$$
\begin{aligned}
-\beta_{i 1} & \frac{\varphi\left(b_{i}\right) P\left(K_{i}\right)+b_{i}}{\left(1+\beta_{i 1}\right) P\left(K_{i}\right)}\left(\frac{b_{i}}{c_{i}}\right)^{\beta_{i 1}} \frac{1}{c_{i}}+\frac{P\left(K_{i}\right) \beta_{i 1} \varphi\left(b_{i}\right)-b_{i}}{b_{i} P\left(K_{i}\right)\left(1+\beta_{i 1}\right)} \\
= & -\beta_{i 2} \frac{\varphi\left(b_{i+1}\right) P\left(K_{i+1}\right)+b_{i+1}}{\left(1+\beta_{i 2}\right) P\left(K_{i+1}\right)}\left(\frac{b_{i+1}}{c_{i}}\right)^{\beta_{i 2}} \frac{1}{c_{i}}+\frac{P\left(K_{i+1}\right) \beta_{i 2} \varphi\left(b_{i+1}\right)-b_{i+1}}{b_{i+1} P\left(K_{i+1}\right)\left(1+\beta_{i 2}\right)}
\end{aligned}
$$

to obtain the volatility.

Theorem 3. Assume that $n$ strike prices $K_{1}, \ldots, K_{n}$ and the corresponding prices of perpetual put options $P\left(K_{1}\right), \ldots, P\left(K_{n}\right)$ satisfying the conditions specified in Theorem 2 are given. The piecewise constant volatility that recovers the option prices can be computed by the following procedure.

1. Calculate $c_{i}, b_{i}$, and $\varphi\left(b_{i}\right)$ for each $i$ by (20), (21), (22), and (23). Additionally, let $b_{1}=c_{1} / 2$ and $b_{n}=x_{0}$.

2. Substitute the numbers computed above into (25) and (26) to obtain $\beta_{i 1}$ and $\beta_{i 2}$ for $i \in\{1, \ldots, n-1\}$. The tail volatility $\beta^{*}=x_{0} / P\left(K_{n}\right)$, and let $\beta_{0}=\beta_{11}$.

Then the piecewise constant volatility that recovers the given option prices is given by

$$
\sigma(x)= \begin{cases}\sqrt{\frac{2 r}{\beta_{0}}}, & 0 \leq x<b_{1}, \\ \sqrt{\frac{2 r}{\beta_{i 1}}}, & b_{i} \leq x<c_{i} \text { for } 1 \leq i \leq n-1, \\ \sqrt{\frac{2 r}{\beta_{i 2}}}, & c_{i} \leq x<b_{i+1} \text { for } 1 \leq i \leq n-1, \\ \sqrt{\frac{2 r}{\beta^{*}}}, & x_{0} \leq x .\end{cases}
$$


Remark 1. Note that the volatility model described above is not the unique piecewise constant volatility model that reproduces the option data. In fact, the choice of the break points $b_{i}$ and $c_{i}$ is arbitrary to some extent. For example, our assumption that the tangent point where $\varphi$ touches the option line is located in the middle of two intersections could easily be changed, thereby giving rise to a different volatility. Also, note that we obtain $2 n$ constant volatilities from $n$ option prices, so the degree of freedom is $n$. However, it seems difficult for us to both decrease the degree of freedom and at the same time ensure the solvability of the problem.

It might happen that $K_{n}$ satisfying $P\left(K_{n}\right)=K_{n}-x_{0}$ cannot be observed from the market. In such a case, to apply the calibration method described above, we must make up a proper $K_{n}$ with $P\left(K_{n}\right)=K_{n}-x_{0}$ using the given option data $P\left(K_{1}\right), \ldots, P\left(K_{n-1}\right)$. Since $P(K)$ is strictly convex with respect to $K$, the strike price $K_{n}$ has to satisfy

$$
K_{n}-x_{0}>P\left(K_{n-1}\right)+\left(K_{n}-K_{n-1}\right) \frac{P\left(K_{n-1}\right)-P\left(K_{n-2}\right)}{K_{n-1}-K_{n-2}} .
$$

There are many ways of choosing $K_{n}$ that satisfies (27). For example, one way would be to use a second-order Taylor expansion of $P$ at the point $K_{n-1}$. However, we omit the details of this procedure.

\section{Numerical illustration}

In the numerical test, we assume that option data observed in the market are actually calculated using a CEV model with $\sigma(x)=x^{-1 / 2}$. Let the current stock price be $x_{0}=10$ and the interest rate be $r=0.1$. Then the $\varphi$ function (cf. [2, pp. 18-19]) is given by

$$
\varphi(x)=26.6423 x \int_{x}^{\infty} \frac{1}{y^{2}} \mathrm{e}^{-0.2 y} \mathrm{~d} y .
$$

According to (3) and (28), we obtain $\hat{K}=13.8778$. A series of strike prices $\left\{K_{i}\right\}$ between 3 and 13.8778 are selected as option data, and the corresponding series of option prices $\left\{P\left(K_{i}\right)\right\}$ can be computed by (9) and (10). The volatility (solid line) produced by Theorem 3 is plotted and compared to the real volatility (dotted line) in Figure 5. As the number of option prices given increases, our predicted volatility fits better and better with the given volatility.

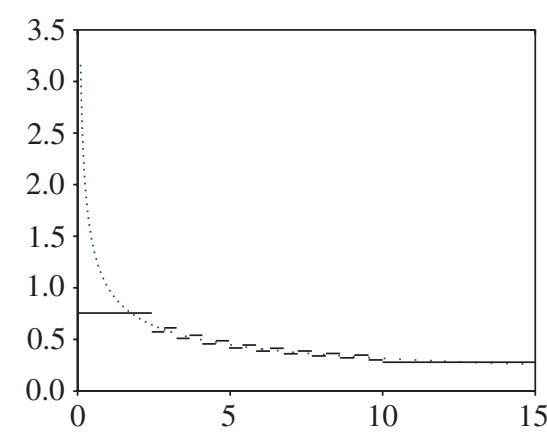

(a) 10 pairs of option data

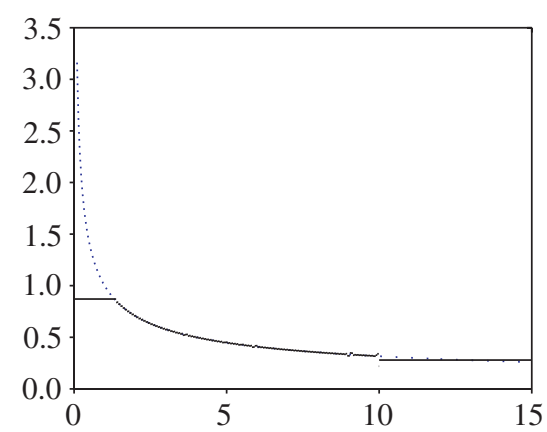

(b) 100 pairs of option data

FIGURE 5: The dotted line is the real volatility $\sigma(x)=x^{-1 / 2}$, and the solid line is the computed volatility. 


\section{References}

[1] Alfonsi, A. And Jourdain, B. (2009). Exact volatility calibration based on a Dupire-type Call-Put duality for perpetual American options. Nonlinear Differential Equations Appl. 16, 523-554.

[2] Borodin, A. N. And Salminen, P. (2002). Handbook of Brownian Motion-Facts and Formulae, 2nd edn. Birkhäuser, Basel.

[3] DupIRe, B. (1994). Pricing with a smile. Risk 7, 18-20.

[4] Ekström, E. AND Hobson, D. (2009). Recovering a time-homogeneous stock price process from perpetual option prices. Preprint. Available at http://arxiv.org/abs/0903.4833v1. 